\title{
Perioperative haemodynamic monitoring by oesophageal Doppler improves outcome of patients with abdominal aortic aneurysm repair
}

\author{
Hillova Mannova $\mathrm{J}^{1}$, Silhart $\mathrm{Z}^{2}$, Sevcik $\mathrm{P}^{1}$, Prokes $\mathrm{A}^{3}$ \\ Department of Anaesthesiology and Intensive Care, Faculty Hospital Brno, Masaryk University, Czech Republic. \\ hillovajitka@seznam.cz
}

\begin{abstract}
Background: AAA repair is associated with high rate of mortality and morbidity. Oesophageal Doppler (OED) can offer a less invasive cost-effective tool for intraoperative monitoring of haemodynamic changes. The aim of the study was to confirm the benefits of haemodynamic optimisation in patients undergoing AAA repair using OED monitoring. We assumed that haemodynamic parameters of patients with OED would be better optimised; they would probably get more fluids during the operation, and their ICU and hospital stay would be shorter as a result of having fewer postoperative complications and lower mortality.

Methods: Seventy patients (Doppler group) who underwent AAA operation in 2003-2008 were matched with 70 patients (control group) operated on AAA in 1998-2002. OED was used to estimate the cardiac output, its variations and fluid management. The administered fluids, length of ICU and hospital stay, frequency of postoperative complications and mortality were compared in Doppler and control groups (Mann-Whitney test, Fisher's exact test). Results: No statistically significant difference was found in preoperative parameters. The Doppler group was administered significantly more crystalloids $(p<0.001)$, colloids $(p<0.001)$, sum-up of fluids $(4000 \mathrm{ml}$ vs. 3000 $\mathrm{ml} ; p<0.001)$ in the perioperative period. The Doppler group had a lower incidence of major serious complications ( 8 vs. $21 ; p=0.034$ ) and shorter ICU ( 4 vs. 6 days; $p<0.001)$ and hospital stay ( 10 vs. 11 days; $p=0.012$ ). Conclusions: The haemodynamic monitoring by oesophageal Doppler can improve the outcome of patients with AAA repair (fewer major complications, shorter ICU and hospital stay) (Tab. 4, Fig. 4, Ref. 25). Full Text in PDF www.elis.sk. Key words: abdominal aortic aneurysm repair, haemodynamic monitoring, oesophageal doppler, outcome.
\end{abstract}

\begin{abstract}
Abdominal aortic aneurysm (AAA) repair is associated with high rates of perioperative and postoperative mortality and morbidity. Abdominal aortic surgery is often performed in elderly patients who suffer from multiple comorbidities. During the operation, it is necessary to take into account the haemodynamic changes caused by aortic cross-clamping and declamping, as well as large blood loss which must be substituted. Moreover, the surgical patients can be hypovolaemic before the operation, and an inadequate blood loss substitution can aggravate the preoperative hypovolaemia. This together with haemodynamic changes during aortic clamping and declamping can impair the tissue perfusion, decrease the oxygen delivery, and increase the postoperative complications.
\end{abstract}

${ }^{1}$ Department of Anaesthesiology and Intensive Care, Masaryk University, Faculty Hospital Brno, Czech Republic, ${ }^{2}$ Department of Surgery, Masaryk University, Faculty Hospital Brno, Czech Republic, and ${ }^{3}$ Faculty of Electrical Engineering and Communication, Brno University of Technology, Czech Republic

Address for correspondence: J. Hillova Mannova, MD, PhD, Polni 17, CZ-59102 Zdar nad Sazavou 2, Czech Republic.

Phone: +420.604232770

Acknowledgements: This work was supported by the Department of Anaesthesiology and Intensive Care, Faculty Hospital Brno, Brno, Czech Republic. We wish to thank Mgr. Zbynek Bortlicek from the Institute of Biostatistics and Analyses at Masaryk University for the statistical analysis.
The usual heart rate monitoring, invasive blood pressure, central venous pressure and urine output do not have to be sufficient for AAA perioperative management. Therefore, a kind of enhanced haemodynamic monitoring is needed.

Pulmonary artery thermodilution has been a commonly used technique in aortic surgery. Nowadays, the new non-invasive techniques are gaining acceptance among anaesthesiologists. These techniques provide an easier access to cardiac output measurement. Oesophageal Doppler (OED) offers a reliable, relatively non-invasive, cost-effective tool for intraoperative monitoring of haemodynamic changes. Furthermore, learning this technique requires less training than regular echocardiography or pulmonary catheterization (1). Oesophageal Doppler can provide continuous monitoring of cardiac output to optimise the tissue perfusion, blood loss substitution and reduce the inflammatory responses and fluid overload (2-4). The question is whether oesophageal Doppler can be of benefit to patients in aortic surgery and whether it is useful for goal-directed haemodynamic therapy in elective AAA repair, in which oesophageal Doppler is rarely used.

The aim of the study was to confirm the benefits of haemodynamic optimisation in patients undergoing AAA repair using oesophageal Doppler. We assumed that haemodynamic parameters of patients with Doppler monitoring would be optimised; probably get more fluids during the operation, and their ICU and hospital stay 
would be shorter as a result of having fewer postoperative complications and lower mortality in comparison to the control group.

\section{Patients and methods}

The local ethics committee agreement was obtained for this study. Seventy patients who underwent abdominal aortic aneurysm operation with oesophageal Doppler monitoring between May 2003 and May 2008 (Doppler group) were matched with seventy patients operated on AAA in 1998-2002 (control group). The first group of 70 patients (Doppler group) had haemodynamic monitoring by echo-oesophageal Doppler (Hemosonic 100, Arrow, reading, PA) during the operation. The group of 70 patients operated on in the earlier years (control group) did not have any enhanced haemodynamic monitoring in the perioperative period. Both groups of patients were operated on under general anaesthesia using opioids, benzodiazepines, muscle relaxants and volatile anaesthetics. Standard monitoring of heart rate, invasive arterial pressure, central venous pressure and urine output were used in both groups of patients. After intubation in the oesophageal Doppler group, an oesophageal Doppler probe was placed in the oesophagus at a distance of 35-40 cm from the incisor teeth and adjusted until an optimal aortic waveform was visible on the screen. The oesophageal Doppler technique measures the blood flow velocity in the descending aorta by means of the Doppler transducer placed at the tip of a flexible probe. Stroke volume is calculated from the aortic velocity measurements; the area under the maximum aortic velocity envelope (VTI) is calculated as a velocity time integral and represents the stroke distance. Assuming that all red blood cells are moving at the maximum velocity and that aortic crosssectional area (A) is constant during systole, stroke volume (SV) is obtained by multiplying the stroke distance (VTI) by the aortic cross-sectional area A. For the measurement of the aortic crosssectional area, Hemosonic has incorporated a transducer into the probe to measure the instantaneous aortic diameter. Oesophageal Doppler was used to estimate the cardiac output, its variations and fluids management with the aim to optimise haemodynamic parameters in the Doppler group of patients. The fluids given in the perioperative period were titrated according to the stroke volume response.

In both groups, the fluids (crystalloids, colloids) administered during anaesthesia were recorded. The length of ICU and hospital stay was compared, together with frequency of mortality, postoperative complications and major serious complications (myocardial infarction, unstable angina pectoris, congestive heart failure, acute renal failure requiring dialysis, bronchopneumonia with respiratory failure, sepsis with multi-organ dysfunction, haemorrhagic shock, disseminated intravascular coagulopathy).

\section{Statistics}

One hundred and forty patients were analysed and separated in two groups, particularly patients who underwent AAA surgery in 1998-2002 (control group) and those subjected to latter surgery in 2003-2008, namely under oesophageal Doppler monitoring (Doppler group).

Each continuous parameter was statistically calculated, namely median, minimum and maximum. Differences between the control and Doppler groups as to patients' histories were analysed by Fisher's exact test. In addition, the postoperative complications were compared by Fisher's exact test. Nonparametric Mann-Whitney test was used for comparison of summed up amount of crystalloid fluids, length of ICU and hospital stay, as well as blood loss. With respect to data discretion, the comparison of total infused colloids was not performed by nonparametric Mann-Whitney test, but categorically by means of Pearson's chi-square test instead.

\section{Results}

The control group was well matched for age, preoperative comorbidities and physiological scores measured using ASA with the Doppler group. There was no statistically significant difference in these parameters between Doppler and control groups (Tab. 1). All

Tab. 1. Patients` preoperative comorbidities, age, ASA.

\begin{tabular}{|c|c|c|c|c|c|}
\hline \multirow[t]{2}{*}{ Parameters } & \multicolumn{2}{|c|}{ Control group $(n=70)$} & \multicolumn{2}{|c|}{ Doppler group $(n=70)$} & \multirow[t]{2}{*}{ p-value 1} \\
\hline & $\mathrm{n}$ & $\%$ & $\mathrm{n}$ & $\%$ & \\
\hline Age $>70$ years & 38 & 54.3 & 42 & 60 & 0.609 \\
\hline Myocardial infarction & 18 & 25.7 & 19 & 27.1 & 1 \\
\hline Angina pectoris II-III degree & 11 & 15.7 & 15 & 21.4 & 0.515 \\
\hline Ischemic chronic heart disease (ICHS) & 38 & 54.3 & 39 & 55.7 & 1 \\
\hline Chronic congestive heart failure, ejection fraction (EF) $<35 \%$ & 3 & 4.3 & 3 & 4.3 & 1 \\
\hline Arrhythmias (supraventricular, ventricular) & 9 & 12.9 & 11 & 15.7 & 0.81 \\
\hline Chronic renal insufficiency $(\mathrm{CHRI})$ creatinine $>110 \mathrm{umol} / 1$ & 12 & 17.1 & 11 & 15.7 & 1 \\
\hline Chronic obstructive pulmonary disease (COPD) FEV/FVC $0.6-0.45$ & 14 & 20 & 15 & 21.4 & 1 \\
\hline Diabetes mellitus & 16 & 22.9 & 13 & 18.6 & 0.677 \\
\hline Hypertension & 49 & 70 & 49 & 70 & 1 \\
\hline Stroke & 9 & 12.9 & 8 & 11.4 & 1 \\
\hline Obesity & 10 & 14.3 & 11 & 15.7 & 1 \\
\hline ASA II & 16 & 22.9 & 18 & 25.7 & 0.844 \\
\hline Surgical blood loss (median) & 700 & & 675 & & 0.4522 \\
\hline
\end{tabular}

${ }^{1}$ p-value Fisher exact test, ${ }^{2}$ Mann-Whitney test 
Tab. 2. Perioperatively administered fluids.

\begin{tabular}{|c|c|c|c|c|c|}
\hline & $\mathrm{n}$ & Median & Min & Max & p-value ${ }^{2}$ \\
\hline \multicolumn{6}{|c|}{ The sum-up amount of fluids } \\
\hline Control group & 70 & 3000 & 1500 & 5500 & \multirow{2}{*}{$<0.001^{*}$} \\
\hline Doppler group & 70 & 4000 & 3000 & 6000 & \\
\hline \multicolumn{6}{|l|}{ Crystalloids (ml) } \\
\hline Control group & 70 & 2650 & 1500 & 4500 & \multirow{2}{*}{$<0.001 *$} \\
\hline \multirow[t]{3}{*}{ Doppler group } & 70 & 3500 & 2500 & 5500 & \\
\hline & \multicolumn{5}{|c|}{ Colloids (ml) } \\
\hline & $\mathrm{N}$ & $0 \mathrm{ml}$ & $</=500$ & $>500$ & p-value ${ }^{3}$ \\
\hline Control group & 70 & 26 & 35 & 9 & \multirow{2}{*}{$<0.001 *$} \\
\hline Doppler group & 70 & 3 & 53 & 14 & \\
\hline
\end{tabular}

${ }^{2}$ Mann-Whitney test, ${ }^{3}$ Pearson's chi-square test

AAAs were infra-renal and the patients underwent aortic surgery through the retroperitoneal approach while surgical blood loss and intra-operative requirements for heterologous blood transfusion were similar in both groups. No statistically significant difference was found between the Doppler and control groups in the median

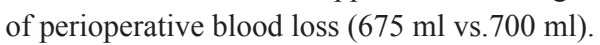

The patients in the Doppler group received significantly more fluids during the perioperative period than the patients in the control group (Tab. 2, Figs 1 and 2). The patients in the Doppler group were given significantly more crystalloids and colloids. Similarly, the summed up amount of fluids was significantly higher in the Doppler group than in the control group (4000 ml vs $3000 \mathrm{ml}$; $\mathrm{p}<0.001)$. When comparing the Doppler group with the control group, the former had shorter mean lengths of ICU stay (4 vs 6 days; $p<0.001)$ and a shorter overall hospital stay (10 vs. 11 days; $\mathrm{p}=0.012$ ) (Tab 3, Figs 3 and 4).

When the frequency of postoperative complications was compared, statistically significant difference was found in the frequency of major serious complications ( 8 vs. 21 complications; $p=0.034$ ). Other complications seemed to be more frequent in the control group but a statistically significant difference was not proved (Tab. 4). In both groups, the most frequent complications were those of cardiovascular origin.

In the Doppler group, significant cardiovascular complications occurred in 19 patients. Seven patients displayed symptoms of cardiac complications, particularly congestive heart failure $(2 \mathrm{x})$, acute myocardial infarction (AMI) (2x), and new haemodynamic significant arrhythmias (atrial, ventricular) (5x). Ten patients had a postoperatively treated hypertension. In the control group, significant haemodynamic complications were more frequent, particularly in 28 cases while cardiac complications were recorded in 21 patients (3x AMI, 3x unstable angina pectoris, $6 \mathrm{x}$ congestive heart failure, $9 \mathrm{x}$ new arrhythmias). One patient was treated for pulmonary embolism and the rest of patients for new postoperative hypertension.

Respiratory complications developed in $8.6 \%(6 / 70)$ of patients in the Doppler group and 10\% (7/70) of patients in the control group. Postoperative respiratory failure, defined as requiring reintubation or ventilation for more than 48 hours postoperatively, was diagnosed in 2 patients in both groups in predisposed individuals (Chronic obstructive pulmonary disease: COPD) as a result of bronchopneumonia.

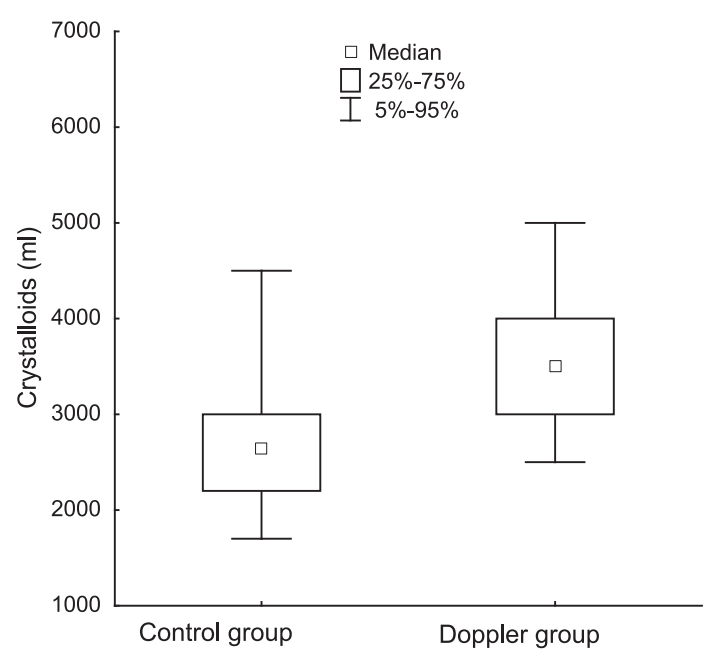

Fig. 1. Comparison of administered crystalloids.

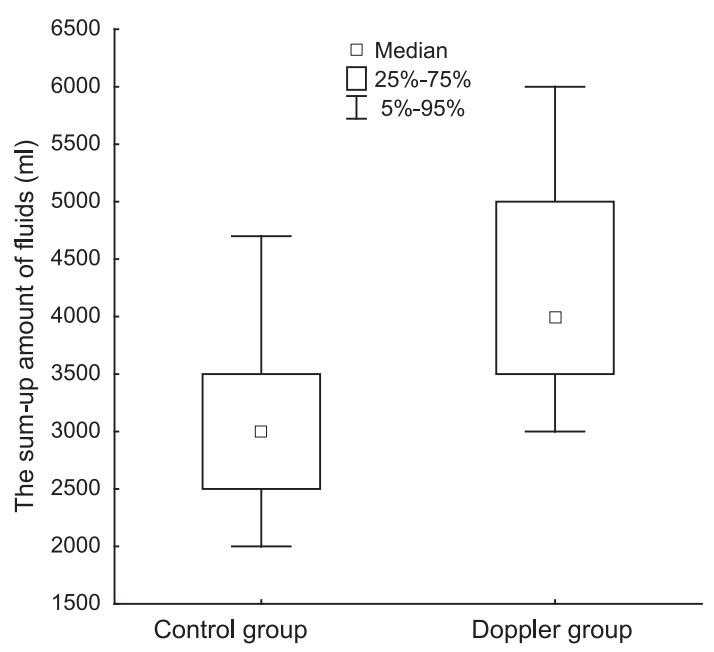

Fig. 2. Comparison of administered fluids (crystalloids + colloids).

Renal complications (renal impairment, acute renal failure ARF, uroinfection) were more frequent in the control group than in the Doppler group (15 in control group vs. 9 in Doppler group). Renal damage manifesting itself as a rise in creatinine level greater than $20 \%$ of the preoperative value, was a complication in 14.3 $\%(10 / 70)$ of patients in the control group and in $11.4 \%(8 / 70)$ of those in the Doppler group. Postoperative renal failure requiring dialysis was recorded in 3 patients $(4.3 \%)$ in the control group. There was no renal failure (0\%) in the Doppler group after the operation.

Surgical complications such as postoperative graft thrombosis, surgical bleeding requiring transfusion or reoperation, superficial wound infection, and prolonged paralytic ileus occurred in 12 patients in the control group and in 7 patients in the Doppler group. Moreover, one patient in the control group suffered from intestinal perforation and necrosis. Deep wound infections and graft infections did not appear. Note that all patients were routinely administered prophylactic antibiotics, usually cephalosporin. 


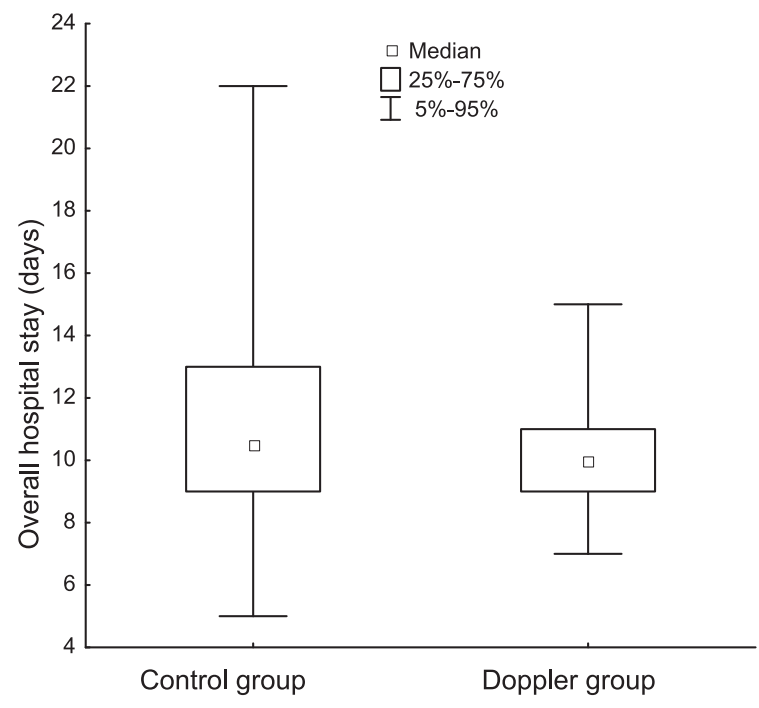

Fig. 3. Overall hospital stay.

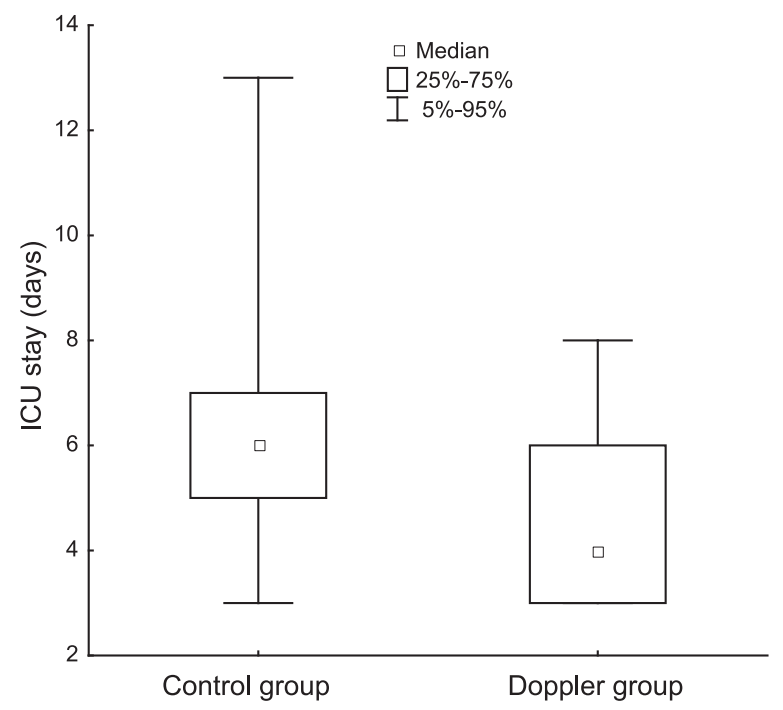

Fig. 4. ICU stay.

Sepsis with multi-organ dysfunction (MODS) was diagnosed in one patient in the Doppler group (urosepsis) and in one patient in the control group (catheter sepsis). Serious postoperative bleeding with haemorrhagic shock and later development of disseminated intravascular coagulopathy (DIC) were noticed in 2 patients in the control group while postoperative coagulopathy (DIC) occurred in 2 patients in the control group and 1 patient in the Doppler group.

Cerebrovascular events appeared in 3 patients in the control group ( $3 \mathrm{x}$ stroke) and in 2 patients in the Doppler group ( $2 \mathrm{x}$ transient ischemic attack - TIA). Paraplegia was a complication in one patient from the control group.

The mortality rate was $7.1 \%(5 / 70)$ in the control group and $1.4 \%(1 / 70)$ in the Doppler group. The percentage of patients who died of cardiac causes was $2.9 \%(2 / 70)$ in the control group and $0 \%$ in the Doppler group. Two patients who died in the con-
Tab. 3. ICU and hospital stay.

\begin{tabular}{lccccc}
\hline & $\mathrm{n}$ & Median & Min & Max & $\mathrm{p}$-value \\
\hline ICU stay & & & & & \\
Control group & 70 & 6 & 2 & 20 & $<0.001^{*}$ \\
Doppler group & 70 & 4 & 2 & 9 & \\
\hline Hospital stay & & & & & \\
Control group & 70 & 11 & 2 & 29 & $0.012^{*}$ \\
Doppler group & 70 & 10 & 5 & 21 & \\
\hline
\end{tabular}

${ }^{2}$ Mann-Whitney test

Tab. 4. Postoperative complications.

\begin{tabular}{|c|c|c|c|c|c|}
\hline \multirow{2}{*}{$\begin{array}{l}\text { Postoperative } \\
\text { Complications }\end{array}$} & \multicolumn{2}{|c|}{ Control group } & \multicolumn{2}{|c|}{ Doppler group } & \multirow[t]{2}{*}{ p-value ${ }^{4}$} \\
\hline & $\mathrm{n}$ & $\%$ & $\mathrm{n}$ & $\%$ & \\
\hline Cardiovascular & 28 & 40 & 19 & 27.1 & 0.152 \\
\hline Respiratory & 7 & 10 & 6 & 8.6 & 1 \\
\hline Renal & 15 & 21.4 & 9 & 12.9 & 0.262 \\
\hline Surgical & 13 & 18.6 & 7 & 10 & 0.227 \\
\hline Others & 11 & 15.7 & 8 & 11.4 & 0.627 \\
\hline Major serious complications & 21 & 30 & 8 & 11.4 & $0.034 *$ \\
\hline Mortality & 5 & 7.1 & 1 & 1.4 & 0.209 \\
\hline
\end{tabular}

${ }^{4}$ Fisher exact test

trol group had preoperative cardiovascular comorbidities (angina pectoris, sick sinus syndrome and hypertension) and suffered from postoperative myocardial infarction on second and fifth days after the operation. As many as $4.3 \%$ of patients $(3 / 70)$ in the control group died of other factors including one case of a haemorrhagic shock (anastomosis, arterial bleeding), one of perforated ileum, and one of multiple-organ failure (ARF, DIC). One predisposed patient (chronic obstructive pulmonary disease, chronic renal insufficiency, and chronic ischemic heart disease) in the Doppler group died of multiple-organ failure (bronchopneumonia followed respiratory failure). Although the mortality rate was higher in the control group, there was no statistically significant difference in mortality rates between the Doppler and control groups.

\section{Discussion}

In our study, we decided for oesophageal Doppler to monitor the haemodynamic parameters. Recently, new users published their reports comparing cardiac output measurements obtained with oesophageal Doppler and thermodilution. Some of these new studies are in agreement with previous conclusions and confirm that OED is a clinically useful alternative to thermodilution. On the other hand, some investigators are still sceptical about the validity of non-invasive measurements $(1,5)$.

Dark MP and Singer M made a systematic search of relevant literature and data synthesis to determine the validity of oesophageal Doppler monitor in measuring the cardiac output in the critically ill. They found eleven validation papers for OED (21 studies) involving 314 patients and 2400 paired measurements and two papers which assessed the validity of Echo-OED as compared with thermodilution. They concluded that the oesophageal Doppler monitoring of changes in cardiac output has high validity (no bias and high clinical agreement with pulmonary artery thermodilution) (6).

However, oesophageal Doppler may not be appropriate for some kinds of surgery since there are limitations concerning its use. 
Firstly, Doppler velocity measurements assume that all erythrocytes travel in the same direction and at the same speed. Secondly, the aortic cross-sectional area is either measured or estimated. Finally, CO calculations assume a constant diversion of blood flow between supra-aortic vessels and the descending aorta. In fact, this proportion is not constant. Aortic surgery is associated with various haemodynamic and cardiac output modifications. These disorders may be partly caused by flow redistribution between supra-aortic and descending aorta regions during clamping and declamping. In the context of this fact, it is in question whether OED monitoring is sufficiently accurate for cardiac output measurement in aortic surgery. Lafanechere et al tried to answer this question in their study (7). Similarly as in our study, they used a new echo-oesophageal Doppler (Hemosonic), which calculates cardiac output from a simultaneous measurement of blood flow velocity and diameter of the descending aorta. In their study, they wanted to find whether this calculation is affected by blood redistribution during aortic clamping. The authors compared cardiac output measured by echooesophageal Doppler and by bolus thermodilution catheter during infra-renal aortic surgery. They confirmed that the bias between both methods was clinically acceptable, and limits of agreement were not significantly modified by aortic clamping. ${ }^{7}$ Their conclusions advocate the use of oesophageal Doppler in our study.

Our results confirmed that oesophageal Doppler could improve the outcome of patients undergoing AAA repair. Patients in the Doppler group were administered more fluids (crystalloid, colloids) in the perioperative period, the postoperative recovery was found to be significantly faster, and the frequency of major serious complications was lower. Our findings are in agreement with the results of other studies comparing the perioperative Dopplerguided intravascular volume replacement strategies with conventional clinical volume replacement in groups of patiens surgically treated for orthopaedic, urologic, gynaecologic, abdominal, and cardiac disorders, as well as those treated for multiple trauma $(8-17)$. In these ten published studies, the investigators used different experimental protocols and all of these studies conclusively report beneficial effects in the Doppler-guided groups $(18,19)$.

For example, a clear benefit after major surgery and hip fracture operations was demonstrated in studies of patients who underwent fluid titration according to stroke volume response using OED. Patients randomised in the optimisation group had fewer complications and shorter hospital stays in comparison with patients treated normally. ${ }^{5}$ Gan et al. and Conway et al. reported improvements after digestive surgery with an earlier ability to drink and eat, less severe complications requiring ICU and shorter hospital stays $(14,15)$.

Sinclair et al. used a protocol to maintain maximal stroke volume and corrected flow time (FTc) in the group of patients undergoing a proximal femoral fracture repair. The protocol group received significantly more fluid and had a higher mean FTc and cardiac output than the control patients despite similar heart rates and blood pressures. Postoperative recovery was found to be faster in the protocol group with the median time being $10 \mathrm{vs} 15$ days (17).

Results similar to our study were obtained in the study carried out by Myethen and Webb. ${ }^{16}$ They studied sixty patients who were randomised to either a control group, receiving treatment according to standard practice, or to a protocol group. The protocol group was given repeated boluses of $6 \%$ hydroxyethyl starch to maximise the OED-measured stroke volume. Compared with the control group, the protocol group had shorter mean lengths of ICU stay (1 vs. 1.7 days) shorter overall hospital stays (6.4 vs 10.1 days, $\mathrm{p}=0.011$ ) and fewer major complications ( 0 vs 6 patients $\mathrm{p}=0.01)(16)$.

In terms of postoperative complications in our study, we confirmed a lower incidence of major serious complications in the Doppler group. The frequency of cardiac complications was lower in the Doppler group than in the control group (Doppler $13 \%$ vs control $30 \%$ ) but no statistically significant difference was proved. It is known that myocardial ischemia remains the most common complication after aortic reconstruction surgery (20). Cardiac complications accounted for $62.5 \%$ of all deaths after AAA repairs (21). In our control group, two patients died of AMI, which accounted for $40 \%$ of all deaths in this group. The rest $(60 \%)$ had other causes (bleeding, MOF, ileum perforation). Whereas in the Doppler group, only one patient died, namely as a result of a non-cardiac event, particularly pneumonia followed by respiratory failure with MODS. Pneumonia counts for one of the most common morbid complications after vascular surgery and its incidence is about $1.5 \%$ with a mortality of $21 \%$. In both of our groups of patients, the second most frequent postoperative complications were renal dysfunctions (control 18.6\%, Doppler $11.4 \%$ ). Renal dysfunction occurs in patients undergoing elective AAA repair but only a small percentage of such patients requires dialysis. Acute renal failure after aortic reconstruction carries a mortality of more than $30 \%(22,23)$. There was no postoperative acute renal failure requiring dialysis in the Doppler group in contrast to three patients with ARF in the control group.

The mortality rate was $7 \%$ in the control group and $1.4 \%$ in the Doppler group. The perioperative mortality rate in the 1960 s for elective AAA repairs was documented to be $14 \%$ to $19 \%$ and has decreased to the current rate of $0-5 \%$ or $1-7 \%$ in leading centres $(24,25)$. Both mortality rates (control and Doppler) correspond with mortality rates in leading centres and it is evident that mortality in the Doppler group was lower than in the control group. However, a statistically significant difference was not shown.

It should be mentioned that no study has been published yet to show the benefit of goal-directed haemodynamic therapy by OED in operations on abdominal aortic aneurysm. On the other hand, this study is limited in that it was not possible to create a prospective randomised study. Ethically, it would be not acceptable to compare a group with haemodynamic monitoring with a group without any enhanced haemodynamic monitoring because paper reviews show benefits from haemodynamic monitoring in patients with high-risk surgery, to which AAA repair indisputably belongs. We could have compared patients with a different kind of haemodynamic monitor technique, particularly thermodilution vs. oesophageal Doppler but in such case we would not get the same results. Therefore, we decided to compare the group monitored with oesophageal Doppler with patients operated on earlier at the end of 1990's when the use of enhanced haemodynamic monitor- 
ing was not spread so much, and recommended for perioperative care. Majority of patients, who underwent AAA repairs at that time, had only standard monitoring, i.e. that of heart rate, blood pressure and urine.

We may say that our control group represents conventional clinical fluid management in AAA repair. In this surgery, it is not possible to use fixed or restrictive fluid regimes because operations of AAA require careful and individual therapeutic considerations strongly related to the perioperative phase, in which fluid therapy plays a major role. While in our control group the fluids were given according to anaesthesiologist assessment and common practice, in OED group oesophageal Doppler enabled haemodynamic optimisation during both phases of aortic clamping and declamping.

In conclusion, we found that oesophageal Doppler is of benefit for patients in elective AAA repair. In the perioperative period, the OED-monitored group was administered significantly more fluid (4000 ml vs $3000 \mathrm{ml} ; \mathrm{p}<0.001)$; both crystalloids $(\mathrm{p}<0.001)$ and colloids $(p<0,001)$. Compared with the control group, the Doppler group had a lower incidence of major serious complications ( 8 vs $21 ; \mathrm{p}=0.034$ ), shorter ICU (4 vs 6 days; $\mathrm{p}<0.001$ ) as well as shorter overall hospital stay ( 10 vs 11 days; $p=0.012$ ). We may conclude that the haemodynamic monitoring by oesophageal Doppler can improve outcomes of patients with AAA repair, nevertheless a confirmation by a prospective randomised study would be much more superior.

\section{References}

1. Choley BP, Singer M. Esophageal Doppler: Noninvasive Cardiac Output Monitor. Echocardiography 2003; 20: 763-769.

2. Abbs SM, Hill AG. Systematic review of the literature for the use of oesophageal Doppler monitor for fluid replacement in major abdominal surgery. Anaesthesia 2008; 63: 44-51.

3. King SL, Lim MST. The Use of the Oesophageal Doppler Monitor in the Intensive Care Unit. Crit Care 2004; 6: 113-122.

4. Kincaid EH, Fly MG, Chang MC. Noninvasive measurements of preload using esophageal Doppler are superior to pressure-based estimates in critically injured patients. Crit Care Med 1999; 27: A111.

5. Cholley BP, Payen D. Noninvasive techniques for measurements of cardiac output. Curr Opin Crit Care 2005; 11: 424-429.

6. Dark PM, Singer M. The validity of trans-esophageal Doppler ultrasonography as a measure of cardiac output in critically ill adults. Intensive Care Med 2004; 30: 2060-2066.

7. Lafanechere A, Albaladejo P, Raux M, Geeraerts T, Bocquet R, Wernet A et al. Cardiac Output Measurement During Infrarenal Aortic Surgery: Echo-Esophageal Doppler versus Thermodilution Catheter. J Cardiothorac Vasc Anesth 2006; 20: 26-30.

8. Noblett SE, Snowden CP, Shenton BK, Horgan AF. Randomized clinical trial assessing the effect of Doppler-optimized fluid management on outcome after elective colorectal resection. Br J Surg 2006; 93: 1069-1076.

9. Wakeling HD, McFall MR, Jenkins CS, Woods WG, Miles WF, Barcaly GR et al. Intraoperative oesophageal Doppler guided fluid management shortens postoperative hospital stay after major bowel surgery. Br J Anaesth 2005; 95: 634-642.
10. McFall MR, Woods WG, Wakelign HG. The use of oesophageal Doppler cardiac output measurement to optimize fluid managementy during colorectal surgery. Eur J Anaesthesiol 2004; 21: 581-583.

11. Mc Kendry M, McGloin H, Saberi D, Caudwell L, Brady AR, Singer M. Randomised controlled trial assessing the impact of a nurse delivered, flow monitored protocol for optimisation of circulatory status after cardiac surgery. BMJ 2004; 329: 258-261.

12. Venn R, Steele A, Richardson P, Poloniecki J, Grounds M, Newman P. Randomized controlled trial to investigate influence of the fluid challange on duration of hospital stay and periopeative morbidity in patients with hip fractures. Br J Anaesth; 2002: 88: 65-71.

13. Chytra I, Pradl R, Bosman R, Pelnar P, Kasal E, Zidkova A. Esophageal Doppler-guided fluid management decreases blood lactate levels in multiple-trauma patients: a randomized controlled trial. Crit Care 2007; 11: R24.

14. Gan TJ, Soppit A, Maroof M, el-Moalem H, Robertson KM, Moretti E et al. Goal-directed intraoperative fluid administration reduces length of hospital stay after major surgery. Anaesthesiology 2002; 97: 820-826.

15. Conway DH, Mayall R, Abdul-Latif MS, Gillian S, Tackaberry C. Randomised controlled trial investigating the influence of intravenous fluid titration using oesophageal Doppler monitoring during bowel surgery. Anaesthesia 2002; 57: 845-849.

16. Mythen MG, Webb AR. Perioperative plasma volume expansion reduces the incidence of gut mucosal hypoperfusion during cardiac surgery. Arch Surg 1995; 130: 423-429.

17. Sincalir S, James S, Singer M. Intraoperative intravascular volume optimization and length of hospital stay after repair of proximal femoral fracture: randomized controlled trial. BMJ 1997; 315: 909-912.

18. Schober P, Loer SA, Schwarte LA. Perioperative Hemodynamic Monitoring with Transeosophageal Doppler Technology. Anaesth Analg 2009; 109: 340-353.

19. Poeze M, Ramseay G, Gerve JW, Singer M. Prediction of postoperative cardiac surgical morbidity and organ failure within 4 hours of intensive care unit admission using esophageal Doppler ultrasonography. Crit Care Med 1999; 27: 1288-1294.

20. Crawford Es, Saleh SH, Babb JW et al. Infrarenal abdominal aortic aneurysm patients: Factor influencing survival after operation performed over a 25-year period. Ann Surg 1981; 193: 699-709.

21. Johnston KW. Multicenter prospective study of nonruptured abdominal aortic aneurysm. Part II Variables predicting morbidity and mortality. J Vasc Surg 1989; 9:437-447.

22. Ostri P, Mouritsen L, Jorgensen B. Renal function following aneurysmectomy of the abdominal aorta. J Cardiovascular Surg 1986; 27: 714-718.

23. Miller DC, Myers BD. Pathophysiology and preventation of acute renal failure associated with thoracoabdominal or abdominal aortic surgery. J Vasc Surg 1987; 5: 518-523.

24. Ghansah Nj, Murphy JT. Complications of Major Aortic and Lower Extremity Vascular Surgery. Sem Cardiothorac Vasc Anesth 2004; 8: $335-361$.

25. Sakalihasan N, Limet R., Defawe O D. Abdominal aortic aneurysm, Lancet 2005, 365: 1677-1689.

Received December 22, 2010. Accepted August 18, 2012. 